INPLASY

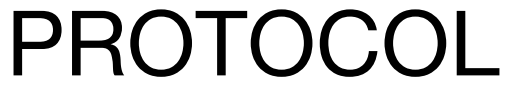

To cite: Li et al. Effect of statin therapy on moderate-to-severe depression: an updated systematic review and metaanalysis. Inplasy protocol 202230016. doi:

10.37766/inplasy2022.3.0016

Received: 03 March 2022

Published: 03 March 2022

Corresponding author:

Peng Li

lipbenzi@126.com

Author Affiliation:

Beijing Institute of

Geriatrics, Beijing Hospital,

National Center of

Gerontology, Institute of

Geriatric Medicine, Chinese

Academy of Medical Sciences.

Support: None.

Review Stage at time of this submission: Data extraction.

Conflicts of interest:

None declared.

\section{Effect of statin therapy on moderate- to-severe depression: an updated systematic review and meta-analysis}

\author{
Li, P1; Liu, J2.
}

Review question / Objective: We aim to assess the antidepressant effects of statin therapy among patients complicated with moderate to severe depression.

Condition being studied: Depression is one of the major causes of disability worldwide, and major depressive disorders (MDD) contribute to a significant heavy disease burden, which is expected to be second by 2050, only to heart disease. Despite great improvement in therapy, the treatment efficacy remains low. Therefore, alternative therapies have been intensely investigated. A substantial body of researches have suggested that inflammation is one of the operative pathways between MDD and increased risk of somatic comorbidities, and some specific depressive symptoms. Depression occurs in most patients with cardiac and cerebrovascular disease due to the long-term effects, and depression increases the risk of cardiovascular disease in the population as a whole and in patients with coronary artery disease or stroke. Several observational studies have demonstrated reduced rates of depression among patients taking statins, which may be related to its anti-inflammatory effect. However, whether statin improves the depressive symptoms and its associated mechanism is still mixed. Furthermore, there is little evidence about statin treatment effect in those with moderate to severe depression. In addition, whether the effect of statin treatment on depressive symptom changes with time or is affected by baseline depression severity or percentage change of lipid levels has not been explored in previous studies.

INPLASY registration number: This protocol was registered with the International Platform of Registered Systematic Review and Meta-Analysis Protocols (INPLASY) on 03 March 2022 and was last updated on 03 March 2022 (registration number INPLASY202230016).

\section{INTRODUCTION}

Review question / Objective: We aim to assess the antidepressant effects of statin therapy among patients complicated with moderate to severe depression. 
Rationale: Several observational studies have demonstrated reduced rates of depression among patients taking statins. However, whether statin improves the depressive symptoms and its associated mechanism is still mixed. Furthermore, there is little evidence about statin treatment effect in those with moderate to severe depression. In addition, whether the effect of statin treatment on depressive symptom changes with time or is affected by baseline depression severity or percentage change of lipid levels has not been explored in previous studies.

Condition being studied: Depression is one of the major causes of disability worldwide, and major depressive disorders (MDD) contribute to a significant heavy disease burden, which is expected to be second by 2050, only to heart disease. Despite great improvement in therapy, the treatment efficacy remains low. Therefore, alternative therapies have been intensely investigated. A substantial body of researches have suggested that inflammation is one of the operative pathways between MDD and increased risk of somatic comorbidities, and some specific depressive symptoms. Depression occurs in most patients with cardiac and cerebrovascular disease due to the long-term effects, and depression increases the risk of cardiovascular disease in the population as a whole and in patients with coronary artery disease or stroke. Several observational studies have demonstrated reduced rates of depression among patients taking statins, which may be related to its anti-inflammatory effect. However, whether statin improves the depressive symptoms and its associated mechanism is still mixed. Furthermore, there is little evidence about statin treatment effect in those with moderate to severe depression. In addition, whether the effect of statin treatment on depressive symptom changes with time or is affected by baseline depression severity or percentage change of lipid levels has not been explored in previous studies.

\section{METHODS}

Search strategy: We undertook an updated meta-analysis and systematic review of randomized clinical trials (RCTs) which were identified in systematic searches of MEDLINE (PUBMED), EMBASE database, Cochrane Controlled Clinical Trials Register Database and the ClinicalTrials.gov website (to December 2021) using terms "statin", "depression", "major depressive disorder", "Hamilton depression rating scale", "remission", "adverse events" and "randomized trial".

Participant or population: Moderate-to severe depression.

Intervention: A statin agent therapy + standard antidepressant drugs.

Comparator: standard antidepressant drugs alone.

Study designs to be included: Randomized controlled trial.

Eligibility criteria: RCTs were considered eligible if they met the following criteria: 1) the research was a prospective RCT; 2) patients were with moderate-to severe depression; 3) the interventions in the statins group consisted of statin agents therapy + standard antidepressant drugs; 4) the control group was treated with standard antidepressant drugs alone; 5) primary endpoint was change of HDRS. Exclusion criteria were (1) patients with mild depression; (2) the interventions in the statins group consisted of statin agents therapy alone; (3) single-arm study; (4) without primary outcome; (5) retrospective study, animal study, case report, or review; and (6) duplicated data.

Information sources: MEDLINE (PUBMED), EMBASE database, Cochrane Controlled Clinical Trials Register Database and the ClinicalTrials.gov website.

Main outcome(s): Primary endpoint was changes of HDRS. 
Additional outcome(s): Secondary endpoints were rate of response to treatment (decrease of HDRS scores by $50 \%$ ), the remission rate and gastrointestinal adverse event.

Data management: All patient's clinical data, including baseline characteristics, interventions, level of lipid, and primary endpoints were extracted by two reviewers (L.P. and L.D.) using a data-collection form.

Quality assessment / Risk of bias analysis: We used the 12 statistic and the $x 2$ test to assess levels of heterogeneity. $12>50 \%$ was referred as significant heterogeneity [14], which indicated that the randomeffects models were used; for all others with non-significant heterogeneity (12 $\leq 50 \%$ ), the fixed-effects models were used. Furthermore, between-study sources of heterogeneity were assessed using subgroup analysis if there was a significant heterogeneity.

Strategy of data synthesis: All results were analyzed with Stata 16.0 software (Stata Corp, Califonia, USA). We used the fixedand random effects (DerSimonian and Laird random-effects) models [13] according to the level of heterogeneity. The relative risk (RR) for dichotomous variables and the standard mean difference (SMD), or weighted mean difference (WMD) for continuous variables were calculated.

Subgroup analysis: In addition, we conducted the prespecified subgroup analysis to examine the effect of statin therapy on HDRS in patients with different baseline HDRS scores. Based on the mean baseline HDRS scores, patients were stratified into low HDRS subgroup (HDRS 525.4) and high HDRS subgroup (HDRS $>25.4)$.

Sensitivity analysis: Sensitivity analyses were performed to determine the effect of individual trial on the overall outcome. We also performed the meta-regression analysis to evaluate the correlation of posttherapeutic or percentage change of lipid values with primary outcome.
Language: English.

Country(ies) involved: China.

Keywords: Depression; Statin; Adverse event; Prognosis; Meta-analysis.

Contributions of each author:

Author 1 - Peng LI - The author drafted the manuscript.

Email: lipbenzi@126.com

Author 2 - Junjun Liu - The author provided statistical expertise.

Email: since8895@126.com 